\title{
Sebaceous carcinoma of the breast predominantly characterized by intraductal growth: a case report
}

\author{
Koichi Ohno ${ }^{1 *} \mathbb{D}$, Toshihiro Okada', Toshitsugu Nakamura² and Hiroshi Koyama ${ }^{3}$
}

\begin{abstract}
Background: Sebaceous carcinoma (SC) is frequently classified as periocular or extraocular. Extraocular SC is rare and mainly occurs in the head and neck, the major salivary glands, or oral mucosa. SC of the breast, lung, and ovary is particularly rare, and the few cases of SC of the breast predominantly exhibit intraductal growth.

Case presentation: A 47-year-old Japanese woman was referred to our hospital with accumulated polymorphic calcification in the left breast which was detected using mammography. Ultrasonography revealed an irregular 13$\mathrm{mm}$ mass in the left breast, and analysis of a core needle biopsy revealed noninvasive ductal carcinoma. Total mastectomy and sentinel lymph node biopsy were performed. Histopathology demonstrated that carcinoma in situ (CIS) represented a significant lesion, and the cytoplasm of tumor cells was clear with numerous minute vacuoles. Immunohistochemical analysis demonstrated that most tumor cells expressed adipophilin. Together, these findings led to a diagnosis of SC, mainly comprising CIS.
\end{abstract}

Conclusions: We encountered a rare case of SC of the breast with predominant CIS.

Keywords: Breast carcinoma, Sebaceous carcinoma, Carcinoma in situ, Immunohistochemistry

\section{Background}

Sebaceous carcinoma (SC) is frequently classified as periocular or extraocular [1]. Extraocular SC is rare, occurring mainly in the head and neck, major salivary glands, oral mucosa, breasts, lungs, and ovaries [2-6]. Although carcinoma in situ (CIS) lesions of SC are rare, particularly associated with extraocular SC, they occur in the head, neck, and upper arm [7-10]. We are unaware of reports of SC of the breast with predominant CIS. For the first time to our knowledge, we report here a case of SC of the breast predominantly characterized by intraductal growth and review the relevant literature.

\section{Case presentation}

A 47-year-old Japanese woman presented with no subjective symptoms. Mammography revealed a clustered pleomorphic calcification in the left $\mathrm{C}$ region (Fig. 1a-d). She was referred to our hospital for further examinations. Her

\footnotetext{
* Correspondence: kohno@suwa.jrc.or.jp

'Department of Breast and Endocrine Surgery, Suwa Red Cross Hospital,

5-11-50, Kogan-douri, Suwa, Nagano 392-8510, Japan

Full list of author information is available at the end of the article
}

medical history stated that her mother had bilateral breast and ovarian cancers. Physical examination did not reveal a mass and papillary secretion in the breasts. The serum levels of the tumor markers carcinoembryonic antigen (CEA), CA15-3, NCC-ST-439, and BCA225 were normal. Ultrasonography identified a solid $(13 \mathrm{~mm} \times 12 \mathrm{~mm} \times 7$ $\mathrm{mm}$ ), irregularly shaped low echo mass with a clear boundary in the left $\mathrm{CD}$ region and a high echo spot in the interior (Fig. 2). The findings of mammography and ultrasonography comprehensively classified the tumor as category 4, according to Breast Imaging Reporting and Data System (BI-RADS) 5th edition. Contrast-enhanced magnetic resonance imaging (MRI) revealed a lesion (14 $\mathrm{mm} \times 11 \mathrm{~mm} \times 12 \mathrm{~mm}$ ) with clear margins in the left $C$ region, confined to the same area (Fig. 3). Analysis of a core needle biopsy of this mass led to a diagnosis of ductal carcinoma in situ (DCIS), nuclear grade $(\mathrm{NG})=2$, which was negative for the expression of estrogen receptor (ER), progesterone receptor (PgR), and human epidermal receptor 2 (HER2). The Ki 67 labeling index was 32.4\%. Positron emission tomography-computed tomography (PET-CT) revealed a breast tumor with a maximum 


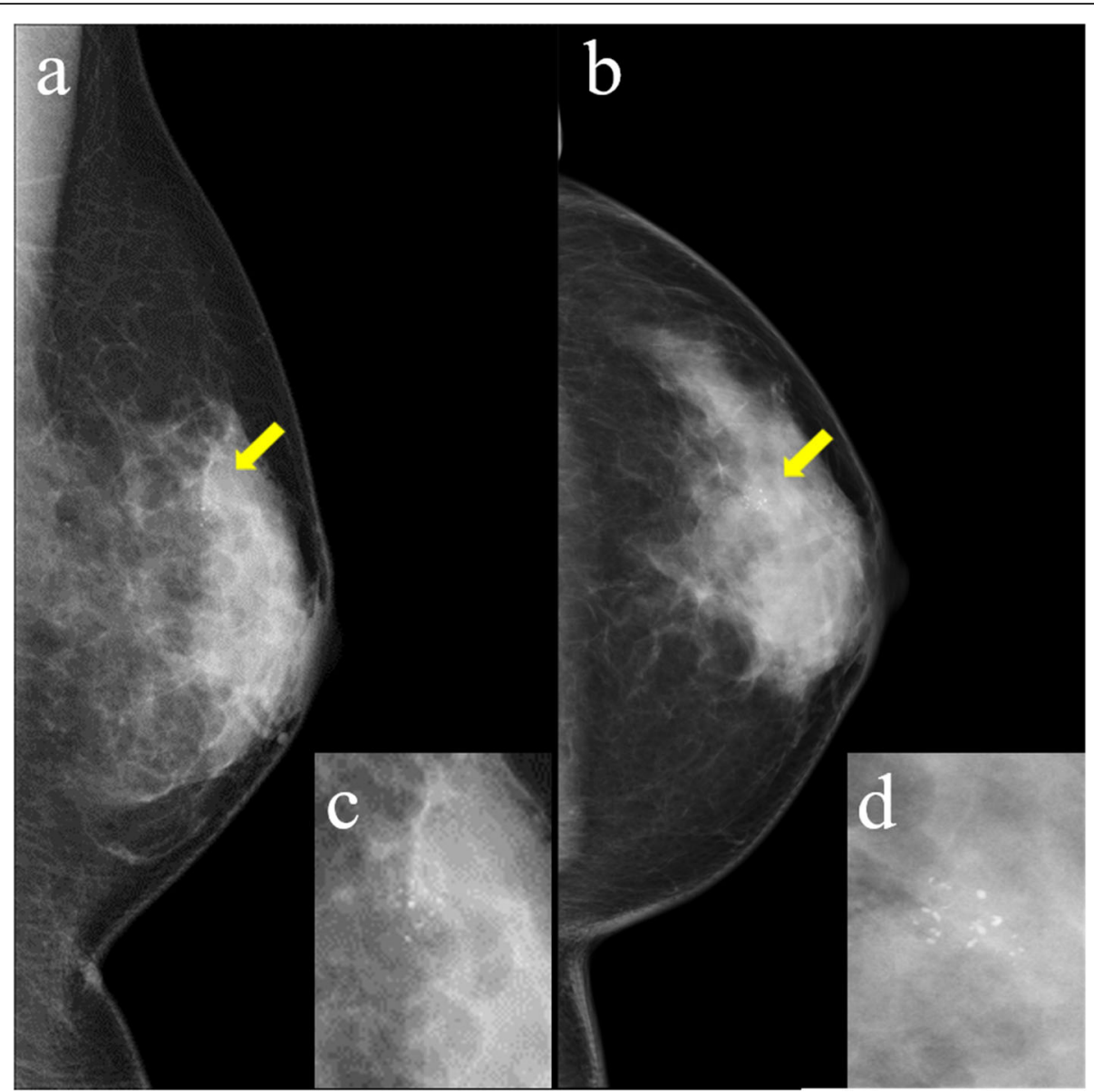

Fig. 1 Mammogram. Mammogram showing clustered pleomorphic calcification in the left MLO-M area (a) and CC-O area (b). c, d Enlargements of the areas indicated by yellow arrows

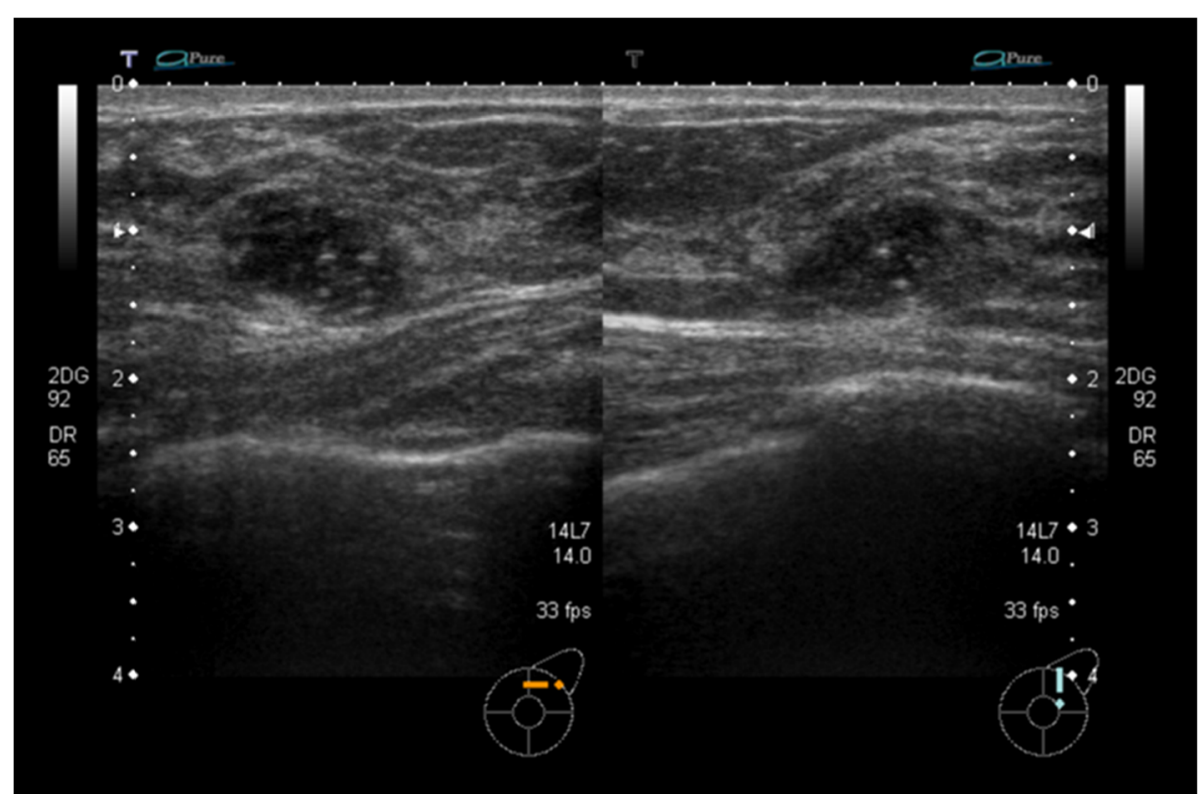

Fig. 2 Ultrasonography. Ultrasonography showing a hypoechoic irregular mass $(13 \mathrm{~mm} \times 12 \mathrm{~mm} \times 7 \mathrm{~mm}$ ) with clear roughening of the boundary and a high echo spot inside in the left CD area 


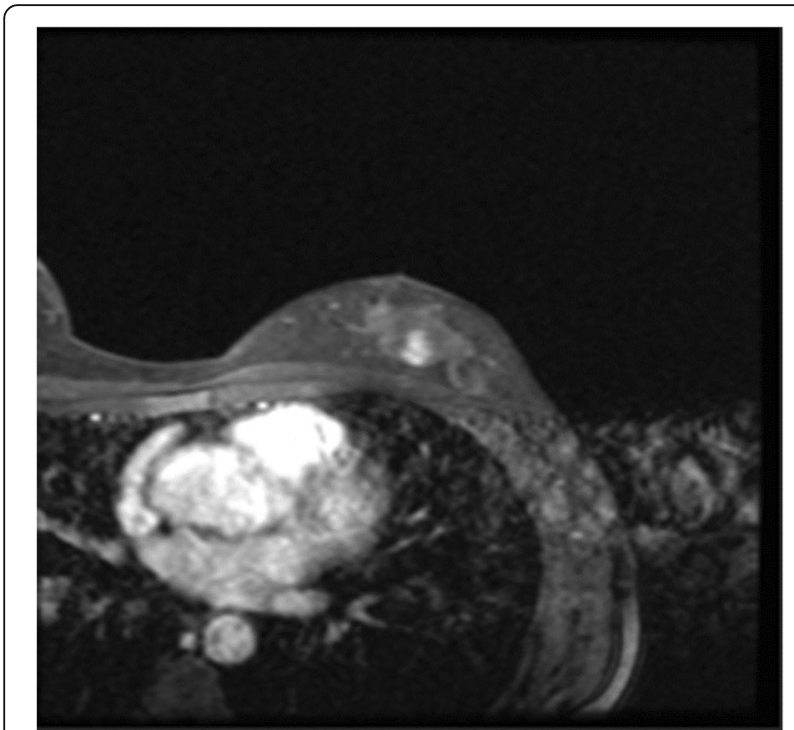

Fig. 3 Contrast-enhanced magnetic resonance imaging (CE-MRI). CE-MRI showing an irregular mass with early arterial enhancement $(14 \mathrm{~mm} \times 11 \mathrm{~mm} \times 12 \mathrm{~mm})$ that was confined to the same area in the left $\mathrm{C}$ area

standardized fluorodeoxyglucose (FDG) uptake value of 3.54 in the left $\mathrm{C}$ region. However, we did not detect an abnormal accumulation of FDG that would indicate metastasis to the axillary lymph nodes, lungs, liver, or bones (Fig. 4).

Although hereditary breast and ovarian cancer syndrome (HBOC) could be considered a possibility because of the patient's age and family history, she did not desire genetic counseling or $B R C A$ testing. Upon the patient's request, we performed total left mastectomy and sentinel lymph node biopsy (SLNB). SLNB was negative for metastasis and therefore axillary lymph node dissection was omitted. Macroscopic findings included a relative clear boundary and a yellowish white mass in the left $C$ region, and continuity with the skin and papilla was not observed (Fig. 5a). Histopathology revealed stromal microinvasion ( $\leq 1-\mathrm{mm}$ diameter) with predominant CIS. Numerous cells exhibited a clear and minute vacuolar cytoplasm, suggesting sebaceous differentiation (Fig. $5 \mathrm{~b}, \mathrm{c}$ ). Immunohistochemical analysis revealed that 50 to $90 \%$ of the tumor cells expressed adipophilin (Fig. 5d, e), leading to a diagnosis of SC of the breast. The CIS lesions comprised tumor cells with similar phenotypes, and comedo necrosis was confirmed. Further tests did not detect venous or lymphatic invasion, $\mathrm{NG}=3$, or expression of ER-, PgR-, or HER2. The Ki 67 labeling index was 90\% (Fig. 5f-j). Lymph node metastasis was not observed. Together, these findings were consistent with pathological T1mi, N0, M0 Stage I. The lesion was mainly CIS with minimal stromal invasion, and we therefore did not offer the patient chemotherapy. Metastasis or recurrence was not observed 16 months after surgery.

\section{Conclusions}

$\mathrm{SC}$ is characterized by lobular forms or nests of tumor cells that exhibit sebaceous differentiation, which distinguishes SC from invasive ductal carcinoma (IDC) [11]. The World Health Organization classifies SC of the breast

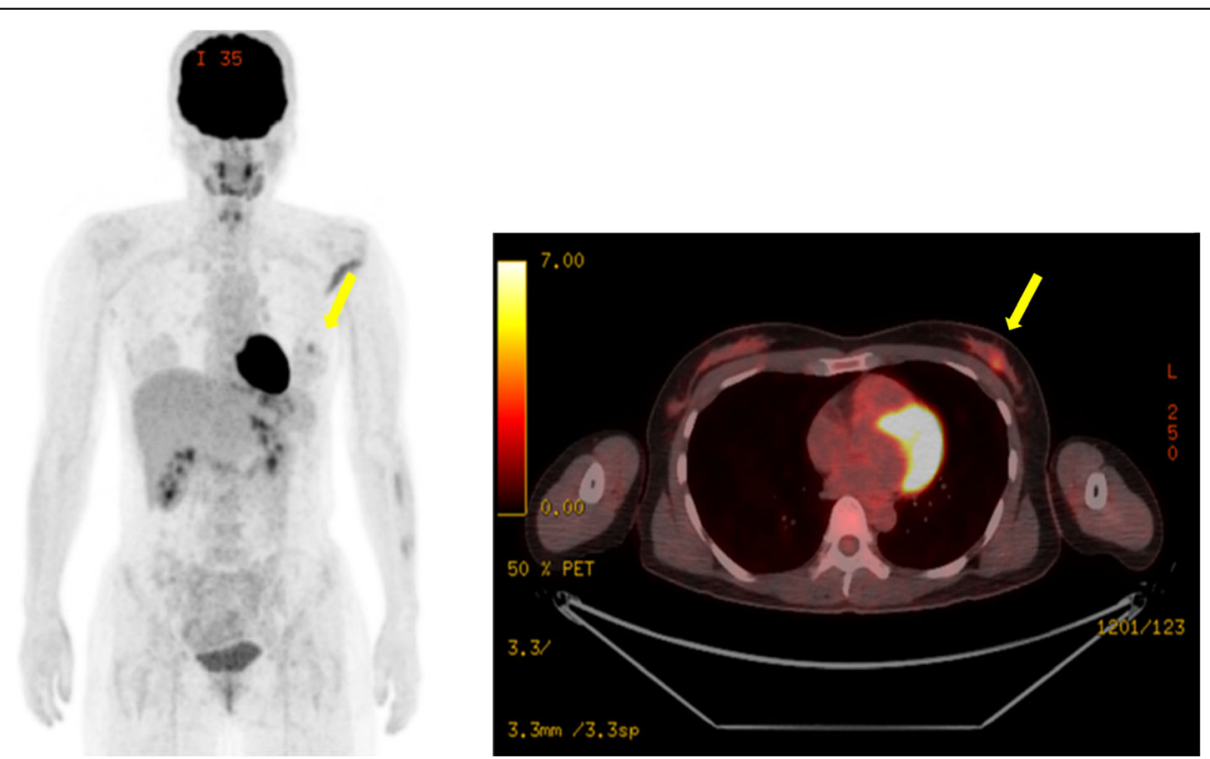

Fig. 4 Whole-body fluorodeoxyglucose (FDG) positron emission tomography-computed tomography (PET-CT). Whole-body FDG PET-CT images showing a mass in the left $C$ area with a maximum standardized uptake value of 3.54 (yellow arrows). Other abnormal accumulations were not detected in other axillary lymph nodes, lungs, liver, and bones 


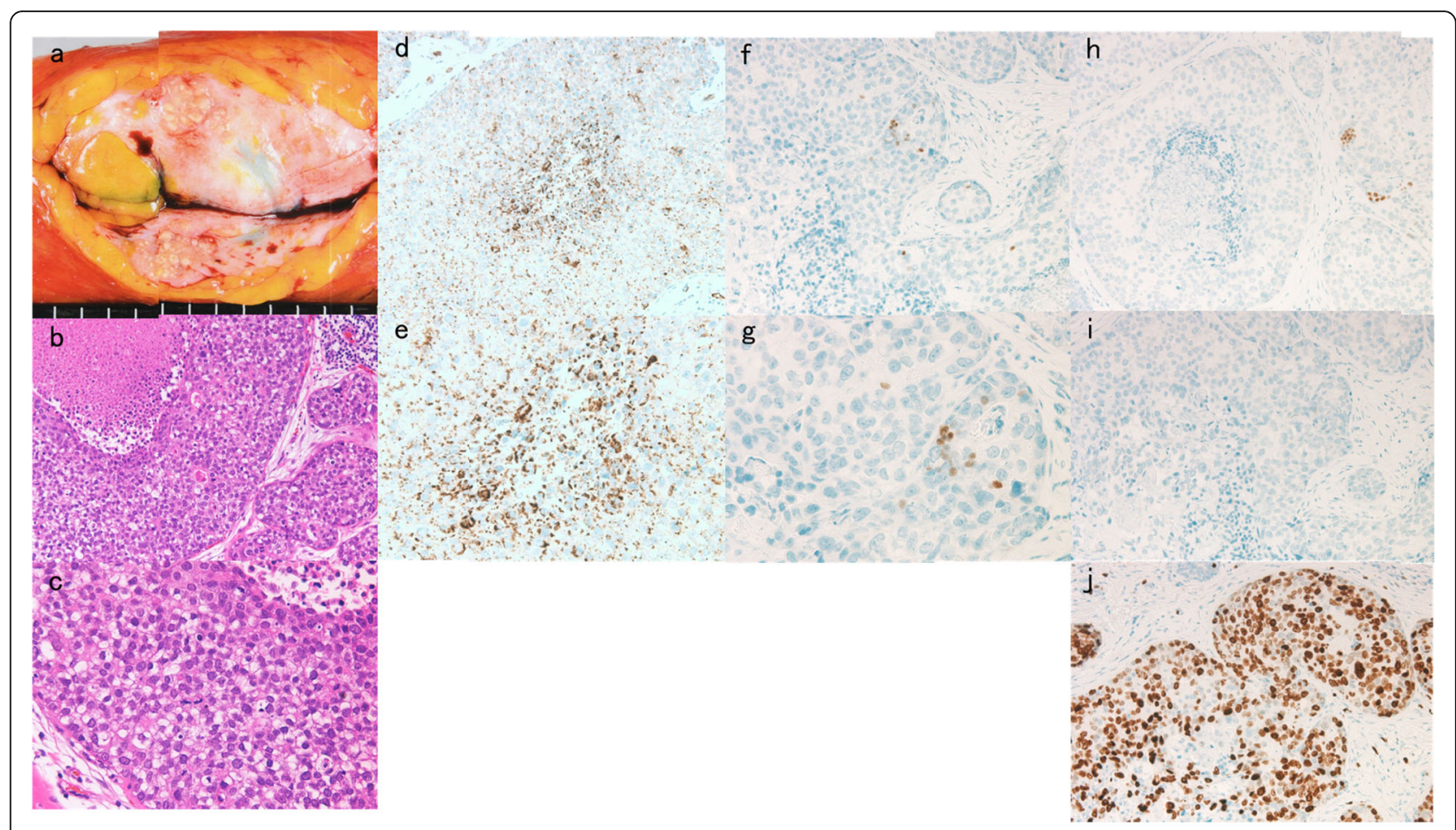

Fig. 5 Macroscopic and pathological findings. a Macroscopic analysis showing relatively clear boundaries. A localized yellowish, white mass was observed, and continuity between the skin and papilla was not evident. b Histopathological analysis revealed carcinoma in situ as the dominant lesion. Hematoxylin and eosin staining revealed cytoplasmic clear and minute vacuolar structures of numerous tumor cells, indicating sebaceous differentiation. $\mathbf{c}$ Higher magnification of $\mathbf{b}$. $\mathbf{d}$ Most tumor cells (50-90\%) were adipophilin-positive. e Higher magnification of $\mathbf{d}$. $\mathbf{f}-\mathbf{i}$ Tumor cells did not detectably express estrogen receptor (ER), f progesterone receptor (PgR) (h), or human epidermal receptor 2 (HER2) (i). $\mathbf{g}$ Higher magnification of $\mathbf{f}$. $\mathbf{j}$ The Ki 67 labeling index was 32.4\%

as primary breast carcinoma if at least half the constituent cells exhibit sebaceous differentiation and are not derived from cutaneous adnexal sebaceous glands as indicated by histopathological analysis [12]. Our present patient's tumor originated deep within the mammary gland and was obviously separated from the adjoining skin. Further, lesions were present in the duct, which is consistent with our diagnosis of $\mathrm{SC}$ of the breast.

Published studies of SC of the breast do not establish its clinical significance. Thus, treatment typically follows that administered to patients with IDC.

Differential diseases of SC of the breast include histologically distinct glycogen-rich clear cell carcinomas and lipid-rich carcinomas. Glycogen-rich clear cell carcinomas typically become periodic acid-Shiff (PAS)positive and therefore can be distinguished from SC cells because they do not produce glycogen [13]. Lipidrich carcinoma is diagnosed according to the presence of tumor cells with a clear cytoplasm comprising at least 90\% lipid-rich vacuoles [12]. Compared with SC of the breast, in which finely vacuolated cells grow into a compact lobulated solid form, the pattern of infiltration of lipid-rich carcinoma is similar to that of typical IDC and with much less conspicuous cellular vacuolization [14]. Thus, diagnosis is achieved through the demonstration of intracytoplasmic lipids detected using Oil Red O or Sudan Black B, or through immunohistochemical detection of the expression of adipophilin [15]. Because of its low incidence, there are no defined immunohistochemical features specific to SC of the breast, except for cytoplasmic lipids. Here, we found numerous clear cells with microvacuolar structures that we suspected to represent sebaceous differentiation. Further, $50-90 \%$ of the tumor cells were adipophilin-positive, which led to our diagnosis of SC of the breast.

Varga et al. [13], Ohara et al. [2], and Numoto et al. [16] propose hypotheses that explain the origin of SC of the breast, respectively, as follows: (a) The mammary glands of patients with breast cancer may exhibit features of sebaceous glands because these glands share the same origin. (b) SC of the breast develops from progenitor cells capable of differentiation into the sebaceous glands within the epithelium. (c) Sebaceous glands are ectopically present in the mammary gland.. If SC of the breast occurs in the absence of other breast cancer pathology, as is the case here, the possibility of scenarios b or c seems plausible. 


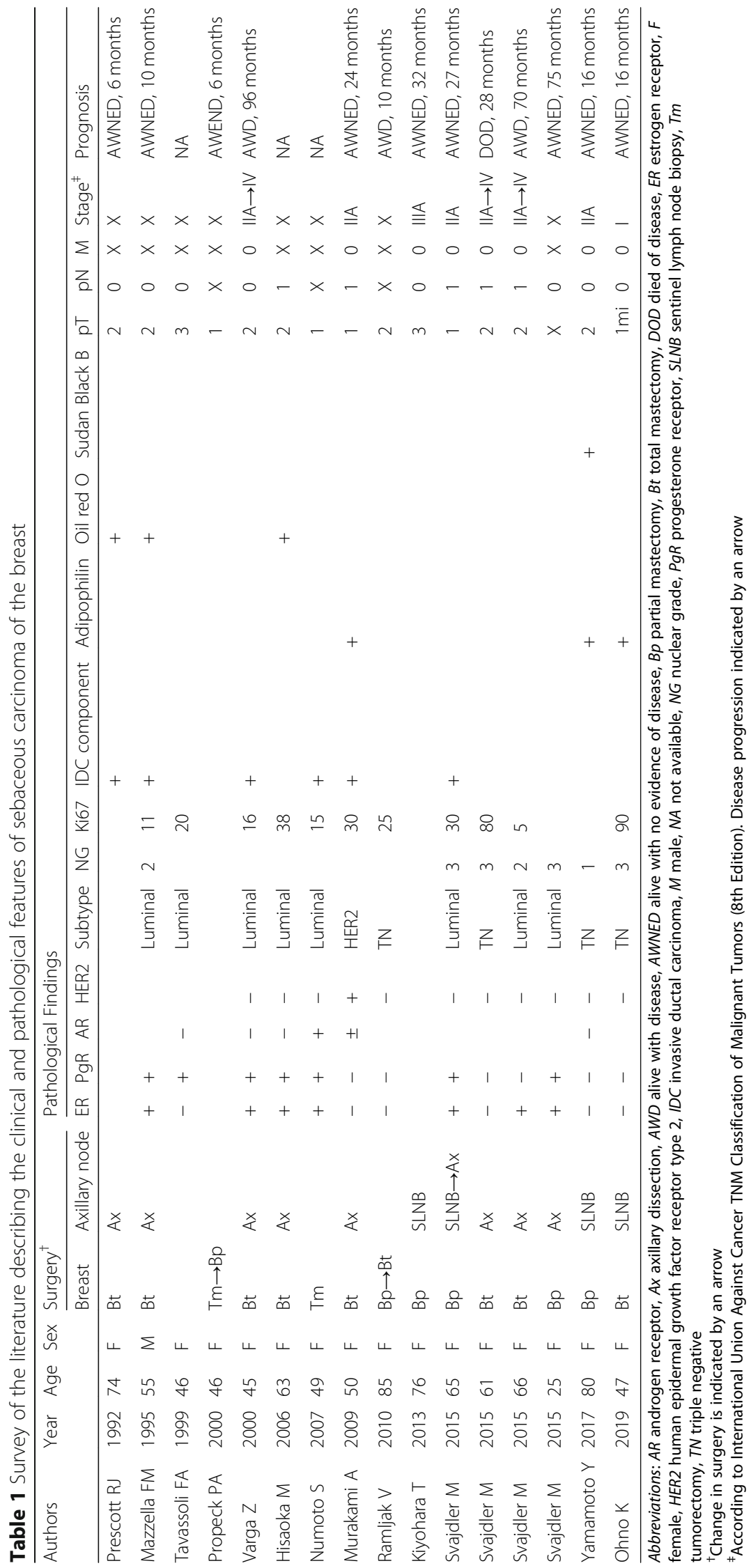


A search of PubMed using the keywords "sebaceous carcinoma AND breast," returned 16 case reports (Table 1) $[4,13,14,16-24]$. These results support our assertion that the present report is the first to identify a patient with pT1mi, N0, M0, Stage I SC of the breast with predominant CIS. As mentioned above, ocular SC is infrequently associated with CIS. Moreover, evidence indicates that CIS is rarely associated with extraocular SC, although extraocular SC occurs in the head and neck and upper arm [7-10]. We identified a fatal case in which metastasis occurred over a relatively short period of 28 months after treatment [14], a case where local recurrence occurred 10 months after partial mastectomy [22], and a case in which skin and bone metastasis occurred 96 months after treatment [13]. Further, we identified a patient who experienced disease-free survival for 75 months after surgery [14]. However, the aspects of our present unique case do not allow us to offer a prognosis. Our current patient was not administered postoperative chemotherapy, and we will therefore conduct a careful follow-up.

In conclusion, here, we encountered a rare case of SC of the breast with predominant CIS. Although our findings contribute to our understanding of the pathogenesis, progression, and prognosis of SC of the breast, further studies of additional cases are required.

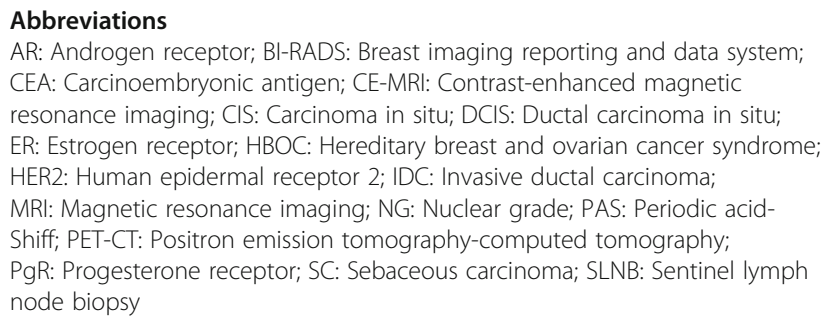

Abbreviations

AR: Androgen receptor; BI-RADS: Breast imaging reporting and data system; CEA: Carcinoembryonic antigen; CE-MRI: Contrast-enhanced magnetic resonance imaging; CIS: Carcinoma in situ; DCIS: Ductal carcinoma in situ; ER: Estrogen receptor; HBOC: Hereditary breast and ovarian cancer syndrome; HER2: Human epidermal receptor 2; IDC: Invasive ductal carcinoma; MRI: Magnetic resonance imaging; NG: Nuclear grade; PAS: Periodic acidShiff; PET-CT: Positron emission tomography-computed tomography; PgR: Progesterone receptor; SC: Sebaceous carcinoma; SLNB: Sentinel lymph node biopsy

\section{Acknowledgements}

We thank Edanz Group (www.edanzediting.com/ac) for editing a draft of this manuscript.

\section{Authors' contributions}

$\mathrm{KO}$ participated in the design of the study and drafted the manuscript. TO, $\mathrm{TN}$, and HK helped to evaluate and interpret the clinical and pathological findings. All authors read and approved the final manuscript.

\section{Funding}

Not applicable

\section{Availability of data and materials}

The dataset supporting the conclusions of this article are available in the manuscript.

\section{Ethics approval and consent to participate}

Not applicable

\section{Consent for publication}

Not applicable

\section{Competing interests}

The authors declare that they have no competing interests.

\section{Author details}

'Department of Breast and Endocrine Surgery, Suwa Red Cross Hospital, 5-11-50, Kogan-douri, Suwa, Nagano 392-8510, Japan. ${ }^{2}$ Department of Diagnostic Pathology, Suwa Red Cross Hospital, 5-11-50, Kogan-douri, Suwa, Nagano 392-8510, Japan. ${ }^{3}$ Koyama Clinic, 1-2557-1, Jonan, Suwa, Nagano 392-0017, Japan.

Received: 27 March 2019 Accepted: 28 January 2020

Published online: 24 February 2020

\section{References}

1. Knackstedt T, Samie FH. Sebaceous carcinoma: a review of the scientific literature. Curr Treat Options Oncol. 2017;18:47.

2. Ohara N, Taguchi K, Yamamoto M, Nagano T, Akagi T. Sebaceous carcinoma of the submandibular gland with high-grade malignancy: report of a case. Pathol. Int. 1998;48:287-91.

3. Siriwardena BS, Tilakaratne WM, Rajapakshe RM. A case of sebaceous carcinoma of the parotid gland. J Oral Pathol Med. 2003;32:121-3.

4. Prescott RJ, Eyden BP, Reeve NL. Sebaceous differentiation in a breast carcinoma with ductal, myoepithelial and squamous elements. Histopathology. 1992;21:181-4.

5. Borczuk AC, Sha KK, Hisler SE, Mann JM, Hajdu SI. Sebaceous carcinoma of the lung: histologic and immunohistochemical characterization of an unusual pulmonary neoplasm: report of a case and review of the literature. Am J Surg Pathol. 2002;26:795-8.

6. Vartanian RK, McRae B, Hessler RB. Sebaceous carcinoma arising in a mature cystic teratoma of the ovary. Int J Gynecol Pathol. 2002;21:418-21.

7. Oka K, Katsumata M. Intraepidermal sebaceous carcinoma: case report. Dermatologica. 1990;180:181-5.

8. Ansai S, Mihara I. Sebaceous carcinoma arising on actinic keratosis. Eur J Dermatol. 2000;10:385-8

9. Ansai S, Takeichi H, Arase S, Kawana S, Kimura T. Sebaceous carcinoma: an immunohistochemical reappraisal. Am J Dermatopathol. 2011;33:579-87.

10. Aung PP, Batrani M, Mirzabeigi M, Goldberg LJ. Extraocular sebaceous carcinoma in situ: report of three cases and review of the literature. J Cutan Pathol. 2014;41:592-6.

11. Yerushalmi R, Hayes MM, Gelmon KA. Breast carcinoma--rare types: review of the literature. Ann Oncol. 2009;20:1763-70.

12. Eusebi V, Ichihara S, Vincent-Salomon A, Sneige N, Sapino A. Exceptionally rare types and variants. In: Lakhani SR, Ellis IO, Schnitt SJ, Tan PH, van de Vijver MJ, editors. WHO classification of tumours of the breast. 4th ed. Lyon: IARC; 2012. p. 71-6.

13. Varga Z, Kolb SA, Flury R, Burkhard R, Caduff R. Sebaceous carcinoma of the breast. Pathol Int. 2000;50:63-6.

14. Svajdler M, Banik P, Poliakova K, Straka L, Hribikova Z, Kinkor Z, et al. Sebaceous carcinoma of the breast: report of four cases and review of the literature. Pol J Pathol. 2015;66:142-8.

15. Ostler DA, Prieto VG, Reed JA, Deavers MT, Lazar AJ, Ivan D. Adipophilin expression in sebaceous tumors and other cutaneous lesions with clear cell histology: an immunohistochemical study of 117 cases. Mod Pathol. 2010; 23:567-73.

16. Numoto S, Iwata J, Nakai T, Aki F. A case of sebaceous carcinoma of the breast. Jpn J Diagn Pathol. 2007;24:58-61 (in Japanese).

17. Mazzella FM, Sieber SC, Braza F. Ductal carcinoma of male breast with prominent lipid-rich component. Pathology. 1995;27:280-3.

18. Tavassoli FA. Sebaceous carcinoma. In: Medina MP, Greenfield S, editors. Pathology of the breast. 2nd ed. Stamford: Appleton and Lange; 1999. p. 555-8.

19. Propeck PA, Warner T, Scanlan KA. Sebaceous carcinoma of the breast in a patient with Muir-Torre syndrome. Am J Roentgenol. 2000;174:541-2.

20. Hisaoka M, Takamatsu Y, Hirano Y, Maeda H, Hamada T. Sebaceous carcinoma of the breast: case report and review of the literature. Virchows Arch. 2006:449:484-8.

21. Murakami A, Kawachi K, Sasaki T, Ishikawa T, Nagashima Y, Nozawa A. Sebaceous carcinoma of the breast. Pathol Int. 2009;59:188-92.

22. Ramljak V, Sarcevic B, Vrdoljak DV, Kelcec IB, Agai M, Ostovic KT. Fine needle aspiration cytology in diagnosing rare breast carcinoma--two case reports. Coll Antropol. 2010;34:201-5.

23. Kiyohara T, Tokuriki A, Kanamori Y, Yasuta M, Imamura Y, Kumakiri M. Superficial sebaceous carcinoma on the areola clinically mimicking mammary Paget's disease. J Dermatol. 2013;40:145-7. 
24. Yamamoto Y, Nakamura T, Koyama H, Kanai T, Moritani S, Ichihara S Sebaceous carcinoma of the breast: a case report. J Surg Case Rep. 2017;3:38.

\section{Publisher's Note}

Springer Nature remains neutral with regard to jurisdictional claims in published maps and institutional affiliations.

Submit your manuscript to a SpringerOpen ${ }^{\mathcal{O}}$ journal and benefit from:

- Convenient online submission

- Rigorous peer review

- Open access: articles freely available online

- High visibility within the field

- Retaining the copyright to your article

Submit your next manuscript at $\boldsymbol{\nabla}$ springeropen.com 